\title{
Non-linear viscoelastic laws for soft biological tissues
}

\author{
Dominique P. Pioletti ${ }^{a, b, 1}$, Lalao R. Rakotomanana ${ }^{a, b}$ \\ ${ }^{a}$ Hôpital Orthopédique de la Suisse Romande, Av. Pierre Decker 4, 1005 Lausanne, Switzerland \\ b Department of Physics, Federal Institute of Technology, 1015 Lausanne, Switzerland
}

(Received 1 November 1999; revised and accepted 7 April 2000)

\begin{abstract}
The development of a conceptual framework to test different viscoelastic constitutive laws is presented. This framework has the advantage of satisfying a priori the thermodynamic restrictions and is valid for large deformations. In addition, the different mechanical contributions are separated according to the time scale of their effects. As an illustration of its ability to model the immediate, short time memory and long time memory contributions, the framework is used to identify mechanical tests performed on human patellar tendons. The resulting viscoelastic law is then proposed to model the soft biological tissues as these tissues present an important viscoelastic behaviour. ( 2000 Éditions scientifiques et médicales Elsevier SAS large deformation / thermodynamics / time scale / biological tissues
\end{abstract}

\section{Introduction}

The soft biological tissues (skin, tendon, ligament) play an important role in the mechanical integrity of the body. Indeed, these tissues have the following functions: to protect the body for the skin, to transfer loads between bones for the ligaments, or between muscles and bones for the tendons. The soft biological tissues are mainly made of collagen and elastin proteins, which bring special mechanical properties. The tissues can be stretched $15 \%$ without damage. They also have an important viscous component in their behaviours (Fung, 1993). From an experimental point of view, mechanical tests performed on soft biological tissues illustrate that the stress depends on the strain rate (Danto and Woo, 1993; Haut, 1983; Pioletti et al., 1999) and that the stress decreases when specimens are subjected to a constant strain (Fung, 1993; Lyon et al., 1988; Pioletti, 1997).

The two experimental facts, strain rate effect and stress relaxation, can be discriminated according to the time scale of their effects. Indeed, any internal process within the tissue has in principle a natural time which is defined as the measure of the time needed for the internal process to move to a new equilibrium after a change of the macroscopic external loading. The different mechanical behaviours can then be classified as a function of their natural time scale. The tissue is said to be elastic if the time scale of the observer, defined as the interval between two macroscopic typical observations, is infinitely smaller than any natural time of all internal processes in the tissue. An elastic tissue remembers entirely its previous state. The tissue has a short time memory (sometimes called anelastic, or more correctly finite time memory), if the observer perceives the tissue to return to the equilibrium state with a time delay. The time scale of the observer is of the same order of any natural time of all internal processes. The tissue has a long time memory (sometimes called viscous) if it never returns to its original reference configuration meaning that the time scale of the observer is infinitely greater than any time scale of all internal processes.

\footnotetext{
${ }^{1}$ E-mail: dominique.pioletti@epfl.ch
} 
The classification of a constitutive law can therefore be defined by the comparison of the time scale of the macroscopic observation and the set of the natural times of all internal processes within the tissue. The present work utilizes the basic concept of the time scale for elaborating constitutive laws of biological tissues. In the classification presented, the strain rate dependence corresponds to the short time memory while the stress relaxation corresponds to the long time memory.

The short and long time memories of biological tissues have been generally considered separately. The short time memory has been described either by extending elastic models (Chiba and Komatsu, 1993; Danto and Woo, 1993) or by superposing spring and dashpot elements (Jamison et al., 1968; Sanjeevi, 1982). With the extension of elastic models, the interpretation of the short time memory depends on the elastic model used, while the addition of spring and dashpot elements is difficult to generalize for large deformations. Recently, we proposed an alternative solution using elastic and viscous potentials that describes the short time memory in large deformations and satisfies the principles of thermodynamics (Pioletti et al., 1998). The long time memory has been well described with the linear viscoelasticity theory developed by Coleman and Noll (1961) or the quasi-linear viscoelastic theory of Fung (1993). Incorporation of the strain rate effect (short time memory) in Fung's theory gave good results at low strain rates $(0.06-0.75 \% / \mathrm{s})$ (Haut and Little, 1972) but imprecise results for higher strain rates (up to 10\%/s) (Woo et al., 1981).

Ideally, the short and the long time memories of a soft biological tissue should be described in the same constitutive law to facilitate either the comparison of viscoelastic properties between tissues or the implementation of the constitutive law in a numerical model. Moreover, the resulting constitutive law should be valid for large deformations (e.g. Pioletti et al., 1995) and should satisfy the principles of thermodynamics. The satisfaction of these requirements represents the goal of this study.

We present here the development of a general constitutive law describing, in the same conceptual framework, the elastic, the short and long time memory contributions. The constitutive law will be based on the basic concept of time scale presented above. As an illustration of its ability to describe the mechanical behaviour of soft biological tissues, the viscoelastic law will be identified to mechanical tests performed on human patellar tendons of knee joints.

\section{Viscoelastic constitutive laws}

Three general principles govern the constitutive laws of materials. The principle of determinism, the principle of local action, which asserts that the present stress at a particle is determined by the history of an arbitrarily small neighborhood of that particle, and the principle of material frame-indifference (objectivity), which asserts that the response of a material is the same for all observers (Noll, 1958). For the biological tissues, we suppose that the stress at a given material point depends on the deformation at that point, not only at the actual time $t$ considered, but also at all previous times. A general constitutive law for such tissue is expressed by the well-known functional (Truesdell and Noll, 1992):

$$
\boldsymbol{S}(t)=\boldsymbol{S}_{\mathrm{e}}(\boldsymbol{C}(t))+\mathfrak{I}_{s=0}^{\infty}\{\boldsymbol{G}(t-s) ; \boldsymbol{C}(t)\}
$$

where $S$ is the second Piola-Kirchhoff stress tensor, $\boldsymbol{C}$ is the right Cauchy-Green strain tensor, $\mathfrak{I}$ is a functional representing the history of $\boldsymbol{G}(t-s)=\boldsymbol{C}(t-s)-\boldsymbol{C}(t)$ and $\boldsymbol{S}_{\mathrm{e}}(\boldsymbol{C}(t))$ is an 'equilibrium term'. The stress $\boldsymbol{S}$ and strain $\boldsymbol{C}$ are two symmetric second-order tensors. The notation in (1) means that $\boldsymbol{G}(t-s)$ is a variable and $\boldsymbol{C}(t)$ is a parameter. 


\subsection{Combination of elastic, short and long time memory contributions}

We propose to rearrange the general constitutive law (1) into three parts according to the time scale:

$$
\boldsymbol{S}=\boldsymbol{S}_{\mathrm{e}}(\boldsymbol{C}(t))+\mathfrak{I}_{s=0}^{\delta}\{\boldsymbol{G}(t-s) ; \boldsymbol{C}(t)\}+\mathfrak{I}_{s=\delta}^{\infty}\{\boldsymbol{G}(t-s) ; \boldsymbol{C}(t)\},
$$

where $\delta \sim 0$. Relation (2) is valid only for a linear functional $\mathfrak{I}$, but it does not necessarily mean a linear response of the material. The constitutive law (2) enables us to consider the different mechanical behaviours based on the time scale of their effects. The first term of the right-hand side of (2) is the immediate contribution (elastic behaviour) as it supports the contribution of the deformation at the actual time $t$. The second term of (2) represents the almost immediate contribution, i.e. the short time memory and can be approximated by a differential type material as $s$ is close to zero (Truesdell and Noll, 1992):

$$
\boldsymbol{S}=\boldsymbol{S}_{\mathrm{e}}(\boldsymbol{C}(t))+\boldsymbol{S}_{\mathrm{v}}(\dot{\boldsymbol{C}}(t) ; \boldsymbol{C}(t))+\mathfrak{I}_{s=\delta}^{\infty}\{\boldsymbol{G}(t-s) ; \boldsymbol{C}(t)\}
$$

with $S_{\mathrm{v}}$ the second Piola-Kirchhoff viscous stress tensor and $\dot{\boldsymbol{C}}$ the strain rate tensor.

Finally, the third term of (2) represents the delayed contribution, i.e. the long time memory. The long time memory contribution requires the history of the strain tensor $\boldsymbol{C}$ at all the past times to calculate the stress $\boldsymbol{S}$. To be consistent with the observation that memories are imperfect, the principle of fading memory is introduced: "deformations which occurred in the distant past have a smaller effect on the present forces than have recent deformations" (Coleman and Noll, 1961). Mathematical interpretation of the fading memory principle asserts that constitutive functionals, such as $\mathfrak{I}$, have continuous Fréchet derivatives relative to a particular norm on a space of histories $\boldsymbol{G}(t-s)$. This leads to an integral relation between the stress and the strain in a first order theory, e.g. (Truesdell and Noll, 1992). The third term of (2) then takes the form:

$$
\mathfrak{I}_{s=\delta}^{\infty}\{\boldsymbol{G}(t-s) ; \boldsymbol{C}(t)\}=\int_{\delta}^{\infty} \Sigma(\boldsymbol{G}(t-s), s ; \boldsymbol{C}(t)) \mathrm{d} s .
$$

We propose then the following general viscoelastic description for soft biological tissues:

$$
\boldsymbol{S}=\boldsymbol{S}_{\mathrm{e}}(\boldsymbol{C}(t))+\boldsymbol{S}_{\mathrm{v}}(\dot{\boldsymbol{C}}(t) ; \boldsymbol{C}(t))+\int_{\delta}^{\infty} \Sigma(\boldsymbol{G}(t-s), s ; \boldsymbol{C}(t)) \mathrm{d} s
$$

where the successive terms of the right-hand side of (5) are the different contributions based on the time scale of their effects. This original description has the advantage of separating the different mechanical behaviours which facilitates the identification process, as will be shown below.

\subsection{Thermodynamic restrictions}

Equation (5) is a general constitutive law which takes into account elastic, short and long time memory contributions. In order to satisfy the general principles of physics, this law is further restricted by thermodynamic principles.

If there is no dissipation (elastic process), satisfaction of the thermodynamic principles reduces to:

$$
\boldsymbol{S}_{\mathrm{e}}=2 \rho_{0} \frac{\partial W_{\mathrm{e}}}{\partial \boldsymbol{C}}
$$


where $\rho_{0}$ is the density expressed in the reference state and $W_{\mathrm{e}}$ is an elastic strain energy potential. In classical thermodynamics, the elastic potential is a function of strain and position, e.g. (Coleman, 1964) (in an isothermal process, temperature is a parameter).

In case of short time memory, a similar framework is applied with the use of a dissipative potential $W_{\mathrm{v}}(\dot{\boldsymbol{C}} ; \boldsymbol{C})$ (or equally called pseudo-potential) from which the viscous stress is derived, e.g. (Germain, 1986). Satisfaction of the thermodynamical principles becomes:

$$
\frac{\partial W_{\mathrm{v}}}{\partial \dot{\boldsymbol{C}}}: \dot{\boldsymbol{C}} \geqslant 0 \quad \forall \dot{\boldsymbol{C}} .
$$

This inequality holds true when the potential $W_{\mathrm{v}}$ is convex and the value of $W_{\mathrm{v}}$ is zero when the strain rate is equal to zero, e.g. (Coussy, 1995; Rakotomanana, 1998).

For the materials with long time memory, thermodynamic variables depend on the history of the strain (temperature is not considered in the present case due to the isothermal process). A complete derivation of thermodynamics theory applied to long time memory effects is found in a fundamental paper of Coleman (1964). A general thermodynamic formulation for the long time memory is beyond the scope of the present study. In the particular case where exponential relaxation functions are used, it can be demonstrated that the thermodynamic principles are satisfied, e.g. (Fabrizio and Morro, 1985; Rabotnov, 1977). Moreover, use of exponential for the relaxation function is in agreement with the principle of fading memory as exponential is an influence function of arbitrary order (Coleman and Noll, 1961).

\subsection{Isotropy and homogeneity}

In order to get a tractable identification process, the material is considered to be homogeneous and isotropic. In that case, the elastic potential $W_{\mathrm{e}}$ can be expressed as a function of three invariants of the deformation tensor, e.g. (Boehler, 1987):

$$
W_{\mathrm{e}}=W_{\mathrm{e}}\left(I_{1}, I_{2}, I_{3}\right)
$$

with:

$$
I_{1}=\operatorname{tr} C, \quad I_{2}=\frac{1}{2}\left((\operatorname{tr} C)^{2}-\operatorname{tr} C^{2}\right), \quad I_{3}=\operatorname{det} \boldsymbol{C} .
$$

The general isotropic representation of the viscous potential $W_{\mathrm{v}}$ involves 10 invariants, e.g. (Boehler, 1987):

$$
W_{\mathrm{v}}=W_{\mathrm{v}}\left(I_{1}, I_{2}, I_{3}, J_{1}, J_{2}, J_{3}, J_{4}, J_{5}, J_{6}, J_{7}\right)
$$

with:

$$
\begin{aligned}
& J_{1}=\operatorname{tr} \dot{\boldsymbol{C}}, \quad J_{2}=\operatorname{tr} \dot{\boldsymbol{C}}^{2}, \quad J_{3}=\operatorname{tr} \dot{\boldsymbol{C}}^{3}, \quad J_{4}=\operatorname{tr}(\boldsymbol{C} \dot{\boldsymbol{C}}), \\
& J_{5}=\operatorname{tr}\left(\boldsymbol{C}^{2} \dot{\boldsymbol{C}}\right), \quad J_{6}=\operatorname{tr}\left(\boldsymbol{C} \dot{\boldsymbol{C}}^{2}\right), \quad J_{7}=\operatorname{tr}\left(\boldsymbol{C}^{2} \dot{\boldsymbol{C}}^{2}\right) .
\end{aligned}
$$

A representation with less invariants can be obtained during the identification process. In the isotropic case, the tensorial representation of the function $\Sigma(\boldsymbol{G}(t-s), s ; \boldsymbol{C}(t))$ can be formulated, e.g. (Pipkin and Rivlin, 1961) but they are too cumbersome to be stated in general. As for the viscous potential, particular experimentally accessible representations can be obtained during the identification process. Consequently, the general constitutive law (5) expressed for the case of isotropy may be re-written in term of elastic potential, 
dissipative potential, and history of strain (with $W_{\mathrm{e}}$ given by unit of volume):

$$
\boldsymbol{S}=2 \frac{\partial}{\partial \boldsymbol{C}} W_{\mathrm{e}}\left(I_{1}, I_{2}, I_{3}\right)+\frac{\partial}{\partial \dot{\boldsymbol{C}}} W_{\mathrm{v}}\left(I_{1}, I_{2}, I_{3}, J_{1}, J_{2}, J_{3}, J_{4}, J_{5}, J_{6}, J_{7}\right)+\int_{\delta}^{\infty} \Sigma(\boldsymbol{G}(t-s), s ; \boldsymbol{C}(t)) \mathrm{d} s .
$$

The constitutive law (12), with satisfaction of inequality (7) for $W_{\mathrm{v}}$ and exponential relaxation functions, is an isotropic viscoelastic description compatible with the basic physical requirements.

\subsection{Incompressibility}

Incompressibility is a kinematic constraint. To satisfy this constraint, an arbitrary hydrostatic pressure $-p C^{-1}$ must be added to the general constitutive law. Geometrically, the incompressibility assumption implicates conservation of volume during deformation of the body. Mathematically, this constraint is expressed by the relation $I_{3}=1$. In the case of incompressibility, the general constitutive law (12) reduces to:

$$
\boldsymbol{S}=-p \boldsymbol{C}^{-1}+2\left(\frac{\partial W_{\mathrm{e}}}{\partial I_{1}}+I_{1} \frac{\partial W_{\mathrm{e}}}{\partial I_{2}}\right) I-2 \frac{\partial W_{\mathrm{e}}}{\partial I_{2}} C+\frac{\partial W_{\mathrm{v}}}{\partial \dot{\boldsymbol{C}}}+\int_{\delta}^{\infty} \Sigma(\boldsymbol{G}(t-s), s ; \boldsymbol{C}(t)) \mathrm{d} s .
$$

The constitutive law (13), with verification of inequality (7) for $W_{\mathrm{v}}$ and exponential relaxation functions, is an isotropic incompressible viscoelastic description compatible with the basic physical requirements.

\section{Experimental set-up}

\subsection{Mechanical tests}

A custom made device has been elaborated to perform uniaxial static and dynamic tests on isolated ligaments and tendons at controlled temperature $\left(37^{\circ} \mathrm{C}\right)$ and humidity (100\%) (Pioletti, 1997; Pioletti et al., 1996; Pioletti et al., 1999). The experimental situation corresponded to the isothermal situation assumed in the theory.

\subsubsection{Elastic and short time memory}

Traction tests were performed on each specimen at four strain rates: $0.6,11,17$ and $24 \% / \mathrm{s}$. To assess that the order of the tests did not affect the results, at the end of the tests the specimens were reloaded with the $0.6 \% / \mathrm{s}$ strain rate. When plotted together, the initial and re-loaded stress-strain curves were identical.

\subsubsection{Long time memory}

The specimens were loaded till $5 \%$ of strain and the strain was maintained constant. The stress relaxation was measured for $1800 \mathrm{~s}$. The stress relaxation was normalized with the relation: (stress relaxation at $t) /($ stress relaxation at $t=0)$.

\subsection{Specimens}

The patellar tendons were collected with their proximal and distal bone attachments from fresh frozen Caucasian knees ( 4 males, mean age \pm SE: $73.4 \pm 2.2$ years). The bone insertions were embedded in a synthetic resin and were fixed upon the supporting structure of the experimental set-up. The fibres were aligned along the loading direction. 


\section{Identification}

The identification process consists first to propose mathematical functions for the potentials $W_{\mathrm{e}}, W_{\mathrm{v}}$ and for the tensor-valued function $\Sigma$ and then to determine, with the mechanical tests, the value of the parameters appearing in these mathematical functions. The form of the mathematical functions is restricted by the fact that the identification process must satisfy four conditions to obtain an admissible constitutive law:

(a) $\boldsymbol{S}(\boldsymbol{C}=\boldsymbol{I})=0$,

(b) $S_{22}=0$,

(c) $C_{11}>1 \Rightarrow C_{22}<1$,

(d) correct stress-strain curve fit.

Condition (a) reflects the fact that the reference geometry is stress free. Condition (b) means that the traction tests were performed without lateral forces applied on the specimens (tractions were performed along $S_{11}$ ). Condition (c) is an obvious requirement for a normal material, but could not be verified by a particular constitutive law. Point (d) is a qualitative requirement.

The form of the compressible (12) or incompressible constitutive law (13), based on the time scale of the different contributions, allowed us to identify each part individually. The identification process starts with the elastic contribution.

\subsection{Elastic behaviour (immediate contribution)}

A supplemental condition applies for the elastic potential $W_{\mathrm{e}}$ :

(e) convexity of $W_{\mathrm{e}}$.

Condition (e) is fundamental for the existence and uniqueness of the solution in a boundary-value problem, e.g. (Chen and Han, 1988; Curnier, 1994). The stress-strain curves are dependent of the strain rate. Consequently, the elastic stress-strain curve is a matter of definition. In this study, the elastic stress-strain curve was defined by the curve obtained at the lowest strain rate $(0.6 \% / \mathrm{s})$, where the strain rate effect was verified to be minimal (Pioletti, 1997).

During the identification process, it was impossible to find an elastic potential that fulfilled the five conditions (a) to (e). In the Appendix, we present the tested elastic potentials in case of compressibility with the conditions satisfied and not satisfied. The incompressibility hypothesis given by the constitutive law (13) was then used. In this case, the elastic potential $W_{\text {e }}$ proposed by Veronda and Westmann (1970):

$$
W_{\mathrm{e}}=\frac{\alpha}{2} \exp \left[\beta\left(I_{1}-3\right)\right]-\frac{\alpha \beta}{4}\left(I_{2}-3\right),
$$

where $\alpha$ and $\beta$ are two elastic parameters, fulfilled the five conditions of the identification and closely fitted the non-linear elastic stress-strain curve obtained with the lowest strain rate (figure 1).

\subsection{Short time memory}

For the short time memory, the condition (a) is slightly modified to incorporate the strain rate:

(a) $\boldsymbol{S}(\dot{\boldsymbol{C}}=0 ; \boldsymbol{C}=\boldsymbol{I})=0$.

The condition (e) is also modified:

(e) convexity of the viscous potential $W_{\mathrm{v}}$ in $\dot{\boldsymbol{C}}$ and $W_{\mathrm{v}}(\dot{\boldsymbol{C}}=0 ; \boldsymbol{C})=0$. 


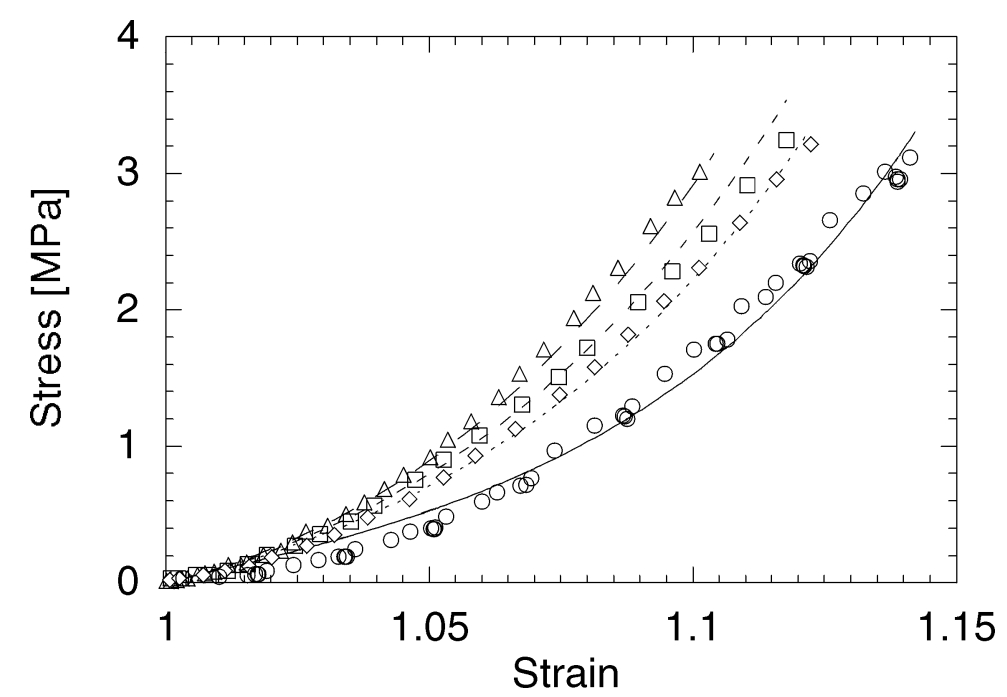

Figure 1. Estimation of the elastic parameters $\alpha$ and $\beta$ and of the viscous parameter $\eta^{\prime}$ using a least square fit of the experimental stress-strain curves obtained at four strain rates for one human patellar tendon specimen. The stress is in the second Piola-Kirchhoff formulation and the strain is in the right

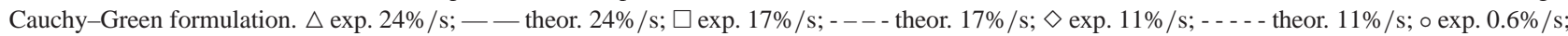

This condition insured that the viscous potential was thermodynamically acceptable. We have recently proposed the following viscous potential which fulfills the five conditions of the identification (Pioletti et al., 1998):

$$
W_{\mathrm{v}}=\frac{\eta^{\prime}}{4} \operatorname{tr}(\dot{\boldsymbol{C}})^{2}\left(I_{1}-3\right)
$$

This potential allowed, with only one parameter $\eta^{\prime}$, to take into account the short time memory (figure 1).

\subsection{Long time memory}

The tensor-valued function $\Sigma$ (equation (13)) is a function of time and strain. For the identification process, the function $\Sigma$ was transformed in two functions of strain $\kappa$ and time $\phi$ only (hypothesis of variables separation):

$$
\Sigma(\boldsymbol{G}(t-s), s ; \boldsymbol{C}(t))=\kappa(\boldsymbol{G}(t-s) ; \boldsymbol{C}(t)) \phi(s) .
$$

The hypothesis of variables separation was recently experimentally verified (Pioletti and Rakotomanana, 2000).

In a relaxation stress identification, the integral part of equation (13) can be written as:

$$
\int_{\delta}^{\infty} \Sigma(\boldsymbol{G}(t-s), s ; \boldsymbol{C}(t)) \mathrm{d} s=\kappa\left(\boldsymbol{G}_{0} ; \boldsymbol{C}_{0}\right) \int_{\delta}^{\infty} \phi(s) \mathrm{d} s,
$$

where $\boldsymbol{G}_{0}$ and $\boldsymbol{C}_{0}$ are constant with respect to the time as the strain is maintained constant. The identification is then performed on the time function:

$$
M(s)=\int_{\delta}^{t} \phi(s) \mathrm{d} s
$$




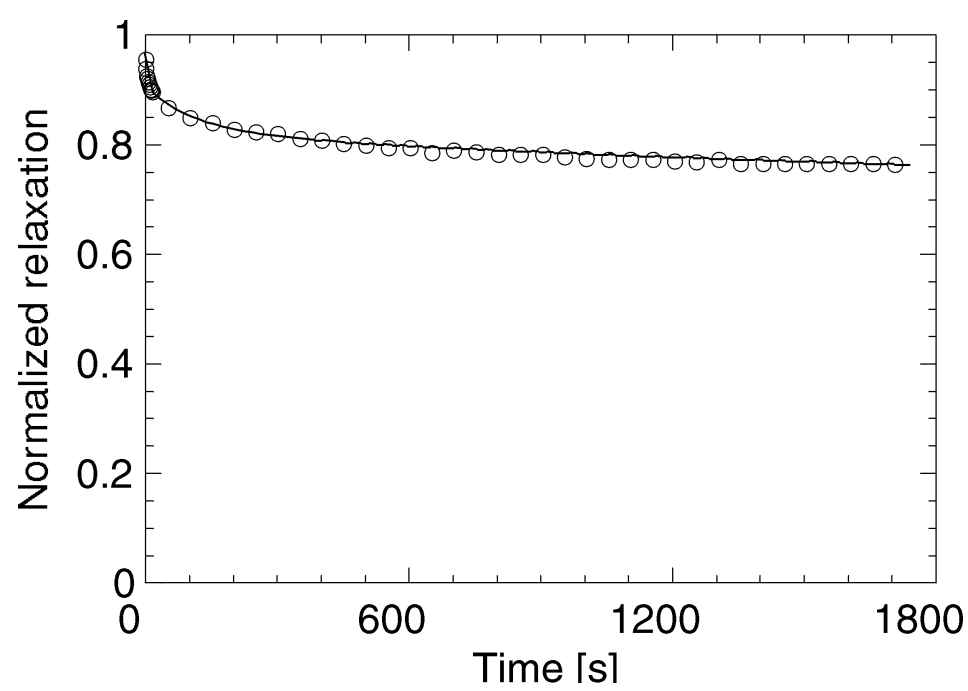

Figure 2. Experimental (dot) and Prony series (plain line) of the normalized relaxation stress in function of time for one human patellar tendon specimen.

The thermodynamic principles are satisfied with the use of a normalized exponential Prony series:

$$
M(s)=\left(\sum_{k=1}^{3} a_{k} \exp \left(-\frac{s}{\tau_{k}}\right)\right) /\left(\sum_{k=1}^{3} a_{k}\right) .
$$

Three exponentials were sufficient to precisely describe the time-relaxation behaviour of the specimens (figure 2).

To take into account the non-linear relationship between the strain and the stress, it has been proposed, e.g. (Findley et al., 1976; Fung, 1993; Lockett, 1972) to use the elastic stress for the function of strain in equation (16):

$$
\kappa(\boldsymbol{G}(t-s) ; \boldsymbol{C}(t))=\boldsymbol{S}_{\mathrm{e}}(\boldsymbol{C}(t-s)) .
$$

The integral part of the constitutive law (13) takes then form:

$$
\int_{\delta}^{t} S_{\mathrm{e}}(\boldsymbol{C}(t-s)) \dot{M}(s) \mathrm{d} s
$$

In summary, the general incompressible constitutive law (13) has the following form after the identification process:

$$
\begin{aligned}
\boldsymbol{S}_{\mathrm{e}} & =-p \boldsymbol{C}^{-1}+\alpha \beta\left(2 \exp \left[\beta\left(I_{1}-3\right)\right]-I_{1}\right) \boldsymbol{I}+\alpha \beta \boldsymbol{C}, \\
\boldsymbol{S}_{\mathrm{v}} & =\eta^{\prime}\left(I_{1}-3\right) \dot{\boldsymbol{C}}, \\
\int_{\delta}^{\infty} \Sigma(\boldsymbol{G}(t-s), s ; \boldsymbol{C}(t)) \mathrm{d} s & =\int_{\delta}^{t} \boldsymbol{S}_{\mathrm{e}}(\boldsymbol{C}(t-s)) \dot{M}(s) \mathrm{d} s,
\end{aligned}
$$

where $M(s)$ is given by (19). The mean values of the viscoelastic parameters appearing in (22) were experimentally obtained and are reported in table $I$.

The originality of the present description is given by the fact that the elasticity, the short and long time memory are described in one framework which satisfies all of the basic physical requirements. Moreover, it is 
Table I. Mean values and \pm SE of the elastic ( $\alpha$ and $\beta$ ), short $\left(\eta^{\prime}\right)$ and long $\left(a_{i}\right.$ and $\left.\tau_{i}\right)$ time memory effect parameters (five specimens).

\begin{tabular}{ccccccccc}
\hline$\alpha$ & $\beta$ & $\eta^{\prime}$ & $a_{1}$ & $a_{2}$ & $a_{3}$ & $\tau_{1}[\mathrm{~s}]$ & $\tau_{2}[\mathrm{~s}]$ & $\tau_{3}[\mathrm{~s}]$ \\
{$[\mathrm{MPa}]$} & & {$[\mathrm{MPas}]$} & & & & & & \\
\hline 0.09 & 66.96 & 438.13 & 0.064 & 0.079 & 0.106 & 7.8 & 156.2 & 1962.3 \\
\pm & \pm & \pm & \pm & \pm & \pm & \pm & \pm & \pm \\
0.02 & 12.99 & 232.20 & 0.005 & 0.008 & 0.017 & 1.8 & 24.0 & 187.9 \\
\hline
\end{tabular}

possible to consider only one behaviour (for example elasticity) independently of the others. This makes the identification process very convenient and facilitates the interpretation of the different mechanical behaviours.

\section{Discussion}

The identification process allowed the mechanical behaviour of the specimens tested to be completely characterized. It was shown that the identification process can not only be reduced to a curve fitting of the experimental data. The five conditions required to obtain a correct identification process reduced the mathematical form of the admissible constitutive laws. In the framework proposed, the resulting constitutive law satisfied the basic physical laws of conservation.

The hypothesis of variables separation in long time memory has been widely used for soft tissues biomechanic, e.g. (Fung, 1993; Johnson et al., 1994; Sauren and Rousseau, 1983; Woo et al., 1981). It has been shown that the relaxation modes showed only slight strain dependences for soft tissues (Soden and Kershaw, 1974). We confirmed this trend for the human patellar tendon specimens (Pioletti and Rakotomanana, 2000).

Use of a Prony series of three exponentials accurately described the time behaviour of the stress relaxation. Identification of exponential functions for the relaxation function is in agreement with the principle of fading memory, e.g. (Coleman and Noll, 1961). Moreover, exponential for the relaxation function is compatible with the principles of thermodynamics.

The compressible identification gave unsatisfactory results during the identification process. Some elastic potentials satisfied the first four conditions but were not convex. These potentials were numerically implemented anyway in a finite element model but furnished no physical results. Therefore, the incompressible hypothesis had to be considered. The incompressible hypothesis has been widely used in soft tissue biomechanics, e.g. (DeHoff et al., 1966; Demiray, 1972; Veronda and Westmann, 1970; Weiss, 1994). The physical motivation behind this assumption is that soft tissues such as ligaments and tendons are mainly composed of water which is nearly incompressible. There is a possibility that some water is expelled from the tissue during the loading procedure, e.g. (Hannafin and Arnoczky, 1994). However, there is actually no clear experimental evidence that the amount of expelled water during traction test is important enough to jeopardize the incompressibility hypothesis.

The implementation of an isotropic constitutive law to model soft tissues which have rather a transverse isotropic behaviour should be performed with caution in numerical simulation. Misleading numerical results could be obtained in situations were multi-axial stresses are present. Indeed, the development of an isotropic constitutive law was imposed by experimental limitations. In view of the lateral specimen size $(10 \mathrm{~mm})$ and the difficulty to have effective clamps on soft tissues, lateral tractions (necessary for determining the extra parameters of a transverse isotropic law) would induce important artefacts rendering the identification process imprecise. The development of transverse isotropic constitutive law would be possible in the theoretical framework proposed. For the elastic potential, five parameters (at least for the linear case) would be needed (Boehler, 1987). For the short and long time memory, a transverse isotropic description would be 
too cumbersome to be generally postulated and the number of parameters to be identified would probably necessitate too many experiments to be feasible.

Formulation of invariant tensor constitutive laws in the presence of irreversible processes such as viscosity or plasticity, is a convenient and powerful method of describing biological tissues properties, e.g. (Cowin, 1995; Rakotomanana et al., 1992). The proposed framework has the ability to describe the short and the long time memory for other soft or hard tissues. The short or the long time memory could be described by identifying different mathematical forms or by using supplementary strain rate invariants in the viscous potential for example. The resulting viscoelastic law will then automatically satisfy the thermodynamic requirements and will be meaningful in large deformations.

\section{Appendix}

Table II. Tested elastic potentials in case of compressibility with the conditions satisfied and not satisfied.

\begin{tabular}{|c|c|c|c|}
\hline Type & Elastic potential & Satisfied & $\begin{array}{c}\text { Not } \\
\text { satisfied }\end{array}$ \\
\hline \multirow[t]{5}{*}{ Poly. } & $W_{\mathrm{e}}=\alpha\left(I_{1}-3\right)+\beta\left(I_{2}-3\right)$ & $\mathrm{a}, \mathrm{b}, \mathrm{c}, \mathrm{e}$ & $\mathrm{d}$ \\
\hline & $W_{\mathrm{e}}=\frac{\alpha}{2}\left(I_{1}-3\right)+\frac{\beta}{2}\left(I_{2}-3\right)-(\alpha+2 \beta)\left(I_{3}-1\right)$ & $\mathrm{a}, \mathrm{b}, \mathrm{c}, \mathrm{d}$ & e \\
\hline & $W_{\mathrm{e}}=\frac{\alpha}{2}\left(I_{1}-3\right)+\frac{\beta}{2}\left(I_{1}-3\right)^{2}+\frac{\gamma}{2}\left(I_{2}-3\right)+\frac{\varepsilon}{2}\left(I_{3}-1\right)$ & $\mathrm{a}, \mathrm{b}$ & d \\
\hline & $W_{\mathrm{e}}=\frac{\alpha}{2}\left(I_{1}-3\right)+\frac{\beta}{2}\left(I_{1}-3\right)^{2}-\frac{\alpha}{2}\left(I_{2}-3\right)$ & $\mathrm{a}, \mathrm{b}$ & d \\
\hline & $W_{\mathrm{e}}=\frac{\alpha}{2}\left(I_{1}-3\right)+\frac{\beta}{2}\left(I_{1}-3\right)^{2}+\frac{\alpha}{2}\left(I_{2}-3\right)$ & $\mathrm{a}, \mathrm{b}$ & d \\
\hline Loga. & $W_{\mathrm{e}}=\frac{\alpha}{2}\left(I_{3}-\log I_{3}\right)+\frac{\beta}{4}\left(I_{1}-3\right)^{2}$ & $\mathrm{a}, \mathrm{b}$ & $\mathrm{d}$ \\
\hline \multirow[t]{9}{*}{ Expo. } & $W_{\mathrm{e}}=\frac{\alpha}{2 \beta}\left\{\operatorname{Exp}\left[\beta\left(I_{1}-3\right)\left(I_{3}-1\right)\right]-1\right\}$ & $\mathrm{a}, \mathrm{b}, \mathrm{c}, \mathrm{d}$ & $\mathrm{e}$ \\
\hline & $W_{\mathrm{e}}=\frac{\alpha}{2 \beta}\left\{\operatorname{Exp}\left[\beta\left(\left(I_{1}-3\right)-\left(I_{3}-1\right)\right)\right]-1\right\}$ & $\mathrm{a}, \mathrm{b}, \mathrm{c}, \mathrm{d}$ & $\mathrm{e}$ \\
\hline & $W_{\mathrm{e}}=\alpha\left\{\operatorname{Exp}\left[\beta\left(\left(I_{1}-3\right)-2\left(I_{2}-3\right)\right)\right]-1\right\}$ & $\mathrm{a}, \mathrm{b}, \mathrm{c}, \mathrm{d}$ & e \\
\hline & $W_{\mathrm{e}}=\frac{\alpha}{2 \beta}\left\{\operatorname{Exp}\left[\beta\left(I_{1}-3\right)\left(I_{2}-3\right)\right]-1\right\}$ & $\mathrm{a}, \mathrm{b}$ & d, e \\
\hline & $W_{\mathrm{e}}=\frac{\alpha}{2}\left\{\operatorname{Exp}\left[\beta\left(I_{1}-3\right)+\gamma\left(I_{2}-3\right)-(\beta+2 \gamma)\left(I_{3}-1\right)\right]-1\right\}$ & $\mathrm{a}, \mathrm{b}, \mathrm{d}$ & $\mathrm{e}$ \\
\hline & $W_{\mathrm{e}}=\alpha \operatorname{Exp}\left[\beta\left(I_{1}-3\right)\right]\left(I_{2}-3\right)$ & & a \\
\hline & $W_{\mathrm{e}}=\alpha \operatorname{Exp}\left[\beta\left(I_{1}-3\right)\right]\left(I_{3}-1\right)$ & & a \\
\hline & $W_{\mathrm{e}}=\frac{\alpha}{2} \operatorname{Exp}\left[\beta\left(I_{1}-3\right)\right]\left\{\left(I_{1}-3\right)-0.5\left(I_{2}-3\right)\right\}$ & $\mathrm{a}, \mathrm{b}, \mathrm{c}, \mathrm{d}$ & e \\
\hline & $W_{\mathrm{e}}=\frac{\alpha}{2} \operatorname{Exp}\left[\beta\left(I_{1}-3\right)\right]\left\{\left(I_{1}-3\right)-\left(I_{3}-1\right)\right\}$ & $\mathrm{a}, \mathrm{b}, \mathrm{c}, \mathrm{d}$ & e \\
\hline \multirow[t]{3}{*}{$\begin{array}{l}\text { LD } \\
\text { Expo. }\end{array}$} & $W_{\mathrm{e}}=\alpha\left\{\tilde{\operatorname{Exp}}\left[\beta\left(I_{1}-3\right)\right]-1\right\}-\frac{\alpha \beta}{2}\left(I_{2}-3\right)$ & $\mathrm{a}, \mathrm{b}, \mathrm{d}, \mathrm{e}$ & $\mathrm{c}$ \\
\hline & $W_{\mathrm{e}}=\alpha\left\{\tilde{\operatorname{Exp}}\left[\beta\left(I_{1}-3\right)\right]-1\right\}-\frac{\alpha \beta}{2}\left(I_{3}-1\right)$ & $a, b, d$ & $\mathrm{c}$ \\
\hline & $W_{\mathrm{e}}=\alpha\left\{\tilde{\operatorname{Exp}}\left[\beta\left(I_{1}-3\right)\right]-1\right\}-\alpha \beta\left(\left(I_{2}-3\right)-\left(I_{3}-1\right)\right)$ & $a, b, c, d$ & $\mathrm{e}$ \\
\hline
\end{tabular}

The notation Exp means that the exponential function is replaced by its third-order limited development (LD).

\section{References}

Boehler J.P., 1987. Applications of Tensor Functions in Solid Mechanics, Springer-Verlag, New York.

Chen W.F., Han D.J., 1988. Plasticity for Structural Engineers, Springer-Verlag, New York. 
Chiba M., Komatsu K., 1993. Mechanical responses of the periodontal ligament in the transverse section of the rat mandibular incisor at various velocities of loading in vitro. J. Biomechanics 26, 561-570.

Coleman B.D., 1964. Thermodynamics of materials with memory. Arch. Rational Mech. Anal. 17, 1-46.

Coleman B.D., Noll W., 1961. Foundations of linear viscoelasticity. Rev. Modern Phys. 3, 239-249.

Coussy O., 1995. Mechanics of Porous Continua, Wiley, New York.

Cowin S.C., 1995. On the minimization and maximization of the strain energy density in cortical bone tissue. J. Biomechanics $28,445-447$.

Curnier A., 1994. Computational Methods in Solid Mechanics, Kluwer Academic Publishers, Boston.

Danto M.I., Woo S.L.Y., 1993. The mechanical properties of skeletally mature rabbit anterior cruciate ligament and patellar tendon over a range of strain rates. J. Orthop. Res. 11, 58-67.

DeHoff P.H., Lianis G., Goldberg W., 1966. An experimental program for finite linear viscoelasticity. Trans. Soc. Rheol. 10, 385-398.

Demiray H., 1972. A note on the elasticity of soft biological tissues. J. Biomechanics 5, 309-311.

Fabrizio M., Morro A., 1985. Thermodynamic restriction on relaxation functions in linear viscoelasticity. Mech. Res. Comm. 12, 101-105.

Findley W.N., Lai J.S., Onaran K., 1976. Creep and Relaxation of Nonlinear Viscoelastic Materials, Dover Publication, New York.

Fung Y.C., 1993. Biomechanics: Mechanical Properties of Living Tissues, Springer-Verlag, New York.

Germain P., 1986. Mécanique-Tome I et II, Ellipses, Paris.

Hannafin J.A., Arnoczky S.P., 1994. Effect of cyclic and static tensile loading on water content and solute diffusion in canine flexor tendons: an in vitro study. J. Orthop. Res. 12, 350-356.

Haut R.C., 1983. Age-dependent influence of strain rate on the tensile failure of rat-tail tendon. ASME J. Biomech. Engng. 105, $296-299$.

Haut R.C., Little R.W., 1972. A constitutive equation for collagen fibers. J. Biomechanics 5, 423-430.

Jamison C.E., Marangoni R.D., Glaser A.A., 1968. Viscoelastic properties of soft tissue by discrete model characterization. J. Biomechanics 1 , $33-46$.

Johnson G.A., Tramaglini D.M., Levine R.E., Ohno K., Choi N.Y., Woo S.L.Y., 1994. Tensile and viscoelastic properties of human patellar tendon. J. Orthop. Res. 12, 796-803.

Lockett F.J., 1972. Nonlinear Viscoelastic Solids, Academic Press, London.

Lyon R.M., Lin H.C., Kwan M.K., Hollis J.M., Akeson W.H., Woo S.L.Y., 1988. Stress relaxation of the anterior cruciate ligament and the patellar tendon. In: Proc. 34th ORS, p. 81.

Noll W., 1958. A mathematical theory of the mechanical of continuous media. Arch. Rational Mech. Anal. 2, 199-226.

Pioletti D.P., 1997. Viscoelastic properties of soft tissues: application to knee ligaments and tendons, PhD thesis, EPF-Lausanne. (http://lgmwww.epfl.ch/staff/pioletti/these.pdf)

Pioletti D.P., Rakotomanana L.R., 2000. On the independence of time and strain effects in the stress relaxation of soft tissues. J. Biomechanics, accepted.

Pioletti D.P., Rakotomanana L.R., Leyvraz P.F., 1999. Strain rate effects on the mechanical behaviour of the anterior cruciate ligament-bone complex. Med. Eng. Phys. 21, 95-100.

Pioletti D.P., Rakotomanana L.R., Benvenuti J.F., Leyvraz P.F., 1998. Viscoelastic constitutive law in large deformations: application to human knee ligaments and tendons. J. Biomechanics 31, 753-757.

Pioletti D.P., Rakotomanana L., Gilliéron C., Leyvraz P.F., Benvenuti J.F., 1996. In: Middletown J. (Ed.), Nonlinear viscoelasticity of the ACL: Experiments and theory, Computer Methods in Biomechanics and Biomedical Engineering, Gordon \& Breach, pp. 271-280.

Pioletti D.P., Heegaard J.H., Rakotomanana R.L., Leyvraz P.F., Blankevoort L., 1995. Experimental and mathematical methods for representing relative surface elongation of the ACL. J. Biomechanics 28, 1123-1126.

Pipkin A.C., Rivlin R.S., 1961. Small deformations superimposed on large deformations in materials with fading memory. ARMA 8, 297-308.

Rabotnov Y.N., 1977. Elements of Hereditary Solid Mechanics, Mir, Moscow.

Rakotomanana L.R., 1998. Contribution à la modélisation géomètrique et thermodynamique d'une classe de milieux faiblement continus. Arch. Rational Mech. Anal. 141, 199-236.

Rakotomanana R.L., Leyvraz P.-F., Curnier A., Heegaard J.H., Rubin P.J., 1992. A finite element model for evaluation of tibial prosthesis-bone interface in total knee replacement. J. Biomechanics 25, 1413-1424.

Sanjeevi R., 1982. A viscoelastic model for the mechanical properties of biological materials. J. Biomechanics 15, 107-109.

Sauren A.A.H.J., Rousseau E.P.M., 1983. A concise sensitivity analysis of the quasi-linear viscoelastic model proposed by Fung. ASME J. Biomech. Engng. 105, 92-95.

Soden P.D., Kershaw I., 1974. Tensile testing of connective tissues. Med. Biol. Eng., 510-518.

Truesdell C., Noll W., 1992. The Non-Linear Field Theories of Mechanics, 2nd ed., Springer-Verlag, New York.

Veronda D.R., Westmann R.A., 1970. Mechanical characterization of skin-finite deformation. J. Biomechanics 3, 111-124.

Weiss J.A., 1994. A constitutive model and finite element representation for transversely isotropic soft tissues, PhD thesis, University of Utah.

Woo S.L.Y., Gomez M.A., Akeson W.H., 1981. The time and history-dependent viscoelastic properties of the canine medial collateral ligaments. ASME J. Biomech. Engng. 103, 293-298. 